\title{
A Game-based Neurofeedback Training System For Cognitive Rehabilitation in the Elderly
}

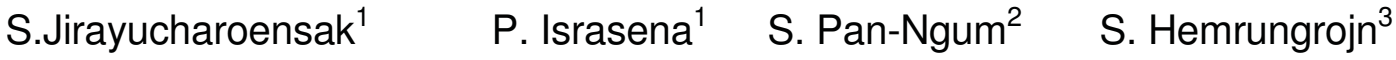 \\ ${ }^{1}$ National Electronics and Computer Technology Center \\ 112 Thailand Science Park, Phahonyothin Road, Klong 1, Klong Luang, Pathumthani 12120, Thailand \\ ${ }^{2}$ Department of Computer Engineering, Faculty of Engineering \\ ${ }^{3}$ Department of Psychiatry, Faculty of Medicine \\ ${ }^{2,3}$ Chulalongkorn University, Bangkok, Thailand \\ suwicha.jirayucharoensak@nectec.or.th, pasin.israsena@nectec.or.th, setha.p@chula.ac.th, solaphat.h@chula.ac.th
}

\begin{abstract}
There is a strong relationship between sustained attention and cognitive performance. The ability to sustain attention potentially leads to enhance cognitive functions. Attention Training provides a promising alternative therapy to enhance cognitive ability and it can be efficiently implemented with Neurofeedback Training (NFT) system. The purpose of this research is to develop a NFT system for attention training to enhance cognitive performance in older adults. The efficiency of the system was evaluated by establishing a clinical trial for healthy elderly and Mild Cognitive Impairment (MCI) patients to perform cognitive rehabilitation. There were totally 24 older adults, age 55-70 yrs, enrolled to this trial. Both healthy and MCI groups performed NFT interventions in 12 half-hour sessions, two sessions a week. Experimental results showed it is possible to train older adults to modify their amplitude of certain brain wave ranges using a neurofeedback protocol targeting several wave ranges for cognitive performance improvement. Most participants succeeded to increase the ratio of their beta/alpha power band in both groups. The relations between specific patterns of EEG activity and levels of cognitive performance can be investigated with the NFT system as a training technique. The system is aimed to encourage older adults to produce specific patterns of cortical activity in connection with an improved level of cognitive performance.
\end{abstract}

\section{Categories and Subject Descriptors}

J.3 [Life and Medical Sciences]: Health

\section{General Terms}

Design, Experimentation.

\section{Keywords}

Cognitive Rehabilitation, Neurofeedback Training System.

\section{INTRODUCTION}

Brain-computer interface $(\mathrm{BCI})$ has been one of the most outstanding biomedical engineering research fields for decades. It provides a technology allowing humans to control external devices by modulating their brain waves. Most BCI applications have been developed for non-invasive brain signal processing

Permission to make digital or hard copies of all or part of this work for personal or classroom use is granted without fee provided that copies are not made or distributed for profit or commercial advantage and that copies bear this notice and the full citation on the first page. To copy otherwise, to republish, to post on servers or to redistribute to lists, requires prior specific permission and/or a fee. REHAB 2014, May 20-23, Oldenburg, Germany Copyright ( 2014 ICST 978-1-63190-011-2 DOI 10.4108/icst.pervasivehealth.2014.255448 which is practical to be implemented in real-world scenarios. There are plenty of successful EEG-based BCI applications such as word speller programs and wheelchair controllers. Not only can BCI be employed to mentally control devices, but it can be implemented for medication and rehabilitation purposes as well. The neurofeedback training system acquires brain waves from an EEG amplifier and then performs feature extractions to classify subject's cognitive states. Subsequently, the system displays the estimated cognition level of the subject through game programs and subjects are able to recognize their own current cognitive state. During training sessions, subjects need to learn how to modulate their brain activity to meet the objective of training system in each session.

NFT system research has undergone considerable proliferation over the years [1]. Most of them focused on improving subject's cognitive state of attention, especially for Attention Deficit Hyperactivity Disorder (ADHD) patients. There is a strong relationship between sustained attention and cognitive performance [2-3]. Attention is the ability to focus on specific information and maintain that focus for the required amount of time. In order for cognitive thought processes to perform, an individual must be able to pay attention to a particular detail of information and fully absorb the material being learned. Consequently, the ability to sustained attention potentially leads to enhance cognitive performance. Attention Training provides a promising alternative method to enhance cognitive ability and it can be implemented with NFT system.

One of the most useful treatments with NFT systems is healing ADHD patients. Several clinical trials successfully treated ADHD children to improve their learning capability and self-control in classroom. A work [4] indicated the NFT approach was as effective as methylphenidate. Also, another research [5] showed the NFT method was superior to a computerized Attention Skills Training (AST). Moreover, several NFT systems were designed to improve cognitive performance in adults and elderly. The purpose of a study [6] is to evaluate the effect of a NFT system on the cognitive performance of healthy elderly. Subjects performed NFT interventions for theta reduction. The results showed the subjects exhibited greater improvement in EEG and behavioral measures. Another work [7] used NFT system to individually enlarge the amplitude of upper alpha frequency band by playing mental rotation games. The result showed 11 of 14 participants significantly improved their cognitive performance compared with non-NFT control group.

The main purpose of the proposed NFT system for attention training is to provide an alternative method for enhancing cognitive performance in older adults by performing game-based NFT interventions. Video game system has been proven to be one 
of the most successful treatments for enhancing cognitive controls and functions in elderly subjects [8]. During training sessions, subjects were entertained by playing games and they were able to play for a long period of time. Subjects need to stay focus on a selective object on the screen. The system estimates the subject's sustained attention level by investigating the power spectrum of beta and alpha frequency bands and then adjusts the characteristics of the displayed object such as racing car speed with the value of attention.

\section{Methodology}

\subsection{Procedure}

A clinical trial was established to evaluate the efficiency of NFT for attention to enhance cognitive performance in healthy elderly and MCI patients. The clinical trial was approved by the Institutional Review Board, Faculty of Medicine, Chulalongkorn University, Bangkok, Thailand. The clinical trial procedure is depicted in Figure 1. First, all of enrolled participants had to take a Montreal Cognitive Assessment (MoCA) test for distinguishing MCI patients from healthy elderly. Second, pre-test score of cognition ability of each subject was measured by Cambridge Neuropsychological Test Automated Battery (CANTAB) test. Subsequently, both healthy and MCI groups performed NFT interventions in 12 sessions, two session a week. Finally, post-test score of cognition ability of each subject was measured by CANTAB. Moreover, EEG analysis was used to monitor their attention and cognitive performance levels.

\subsection{Participants}

The program recruiting announcement was posted on advertisement boards at Chulalongkorn Hospital. Also, a group of healthy adults participating in Thai Red Cross Society's Healthy Elderly Contest were invited to the program. There were totally 24 older adults, age 55-70 yrs, enrolled to this NFT program. Two participants received a very low score and were not allowed to attend the program due to Alzheimer's Disease (AD) possibility. Moreover, two participants in healthy group and one participant in MCI group left the NFT intervention procedure. Consequently, the number of participants completing all training sessions were 8 subjects in healthy group and 11 subjects in MCI group.

\subsection{Montreal Cognitive Assessment}

MoCA [9] was developed to differentiate between normal agerelated cognitive decline and MCI. It has been demonstrated to be valid and reliable for screening of $\mathrm{MCI}$ in various cross-cultural clinical samples. The MoCA assesses several cognitive domains: visuospatial, executive functions, language abilities, attention, memory and orientation. The MoCA has more emphasis on tasks of frontal executive functioning and attention than the TMSE, which may make it more sensitive in detecting non-AD dementia.

\subsection{CANTAB}

CANTAB [10] battery uses touch screen technology to give rapid, accurate, and language independent cognitive assessment. The CANTAB battery now comprises 22 tests to assess different aspects of cognitive functioning, including: 1) tests of shifting and sustained attention, 2) reaction time, 3) visual memory and learning, 4) executive processes such as planning and spatial working memory, and 5) decision making and response control. CANTAB tests have good test-retest reliability and parallel forms of many tests help guard against learning effects over repeated testing sessions. Floor and ceiling effects are avoided by gradually analyzing by comparing the total correct score in each test increasing the demands of the tests. The key outcome measures with the normative data (given by age, gender and/or IQ) or baseline data of individual.

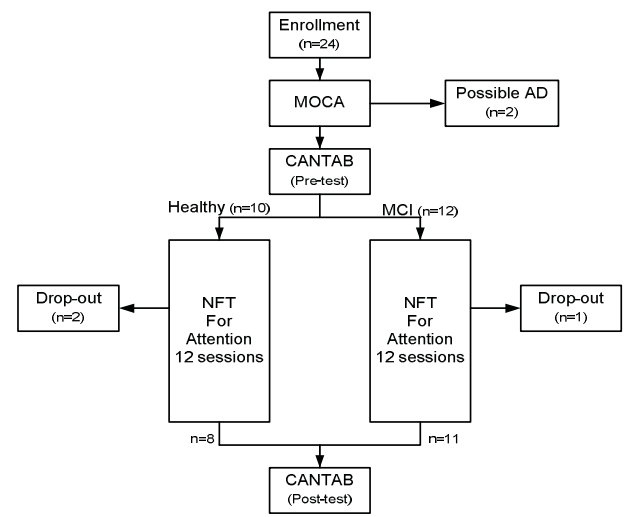

Figure 1. Procedure of the clinical trial

\subsection{Neurofeedback Training System}

The architecture of the proposed NF system for attention training is illustrated in Figure 2. An EEG Amplifier acquires raw EEG signals at $\mathrm{AF} 3, \mathrm{AF} 4, \mathrm{O} 1$ and $\mathrm{O} 2$ with $128 \mathrm{~Hz}$ sampling rate. All of EEG artifacts must be removed from raw brain signals before the system estimates the state of attention. The system exploits Independent Component Analysis (ICA) to remove all of EEG artifacts. The attention level is estimated by investigating the relative power ratio of Beta/Alpha frequency bands. Subsequently, the attention level is used to adjust the characteristic of a target object on the computer screen.

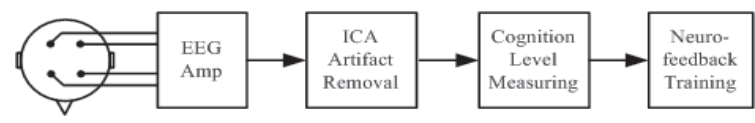

Figure 2. Neurofeedback System for Attention Training

Video game neurofeedback training system is one of the most successful supplementary brain exercise for enhancing cognitive controls and functions in elderly subjects. During training sessions, subjects were entertained by playing games and they were able to play for a long period of time. The more they were trained, the more their cognitive function improvement. Both healthy and MCI groups performed NFT interventions in 12 sessions and a session takes about 30 minutes. In each session, subjects had to perform 6 of 2-minute NFT interventions. There was a 3-minute break for participant's comfort between each game. The NF system for attention training had 6 games, shown in Figure 3. Subjects played one game in 2 consecutive sessions (one week). The last additional brief session $\left(13^{\text {th }}\right.$ session) was to compare the first and last NF intervention with the first game of the training program.

The video game NF system for attention training calculates the subject's sustained attention level by investigating the power spectrum of beta and alpha frequency bands and then adjusts the characteristics of the displayed object such as racing car speed with the attention level. Therefore, subjects were able to recognize their own current cognitive state of attention and attempt to stay focus throughout each training session. During training sessions, subjects needed to learn how to modulate their brain activity to meet the objective of training system in each session.

The ability to sustain attention with distractions offers better attention training efficiency. Our games in the NFT system implemented distractions interrupting the sustained attention of subjects periodically. One of the NFT games called "The 
Museum" added color in a part of picture at a time, if the subject stayed focus on the picture. While watching the picture, the subject would be interrupted by a person walking pass the picture and a person taking a photo. Moreover, subjects performed NFT sessions in a common room in the hospital. During training sessions, they were also interrupted by hospital's staffs entering and exiting the room.
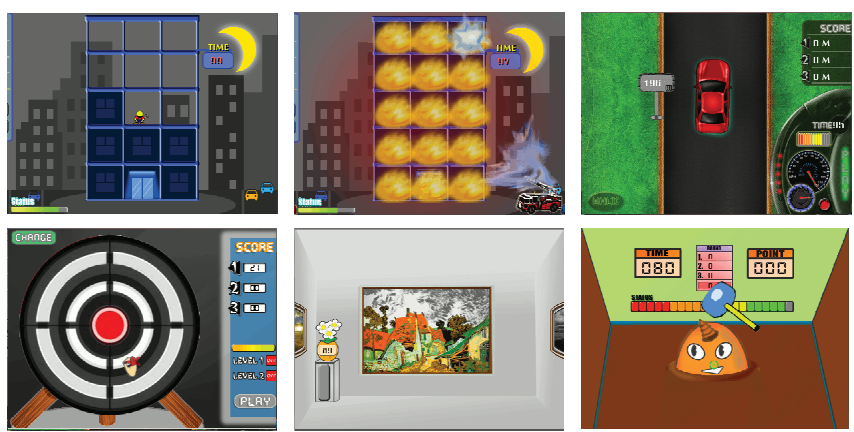

Figure 3. Attention Training Games

\subsection{Attention State Detection}

The state of attention can be identified by detecting lower power spectrum of alpha band and higher power spectrum of beta band simultaneously [11]. The definition of Attention level is

$$
\text { Attention }=\frac{\mathrm{K} \beta * \mathrm{P} \beta}{\mathrm{K} \alpha * \mathrm{P} \alpha}
$$

$\mathrm{K}_{\beta}, \mathrm{K}_{\alpha}$ :Constant for scaling the value of Beta and Alpha band power.

$\mathrm{P}_{\beta}, \mathrm{P}_{\alpha}$ :Relative power spectrum of Beta and Alpha band.

The system acquires EEG signals from the EEG amplifier "Emotiv EPOC" at 4 positions - AF3, AF4, O1 and O2. The amplifier has $128 \mathrm{~Hz}$ sampling rate and its bandpass filter is set to $0.1-60 \mathrm{~Hz}$.The sample block size is assigned to 64 . However, the system utilizes a buffer of 192 previous samples to make the data analysis more accurate. Consequently, at every half a second, the system performs the removal algorithm from these 256 samples of each channel. The mean value of relative power spectrum of the beta and alpha bands measured at AF3, AF4, O1 and $\mathrm{O} 2$ were computed. The triggering threshold was automatically adjusted according to the rate of success of the participant in order to avoid causing a sense of frustration or failure. If the threshold was much higher than current attention level, the program gradually adjusted the threshold lower and vice versa.

\subsection{EEG Artifact Removal Technique}

The cognitive state estimation must be precise and robust enough to provide proper neurofeedback responses in real-time. Electrooculography (EOG) and Electromyography (EMG) artifacts are unavoidable and unpredictable due to subject's physical conditions. This factor makes the cognitive state classifier less reliable. EEG artifacts are mainly constituted of ocular and muscular artifacts. The neurofeedback system for attention training utilizes Independent Component Analysis (ICA) to remove EEG artifacts. ICA is responsible for separating scalp EEG signals into functionally independent brain activities and other non-neural activities or EEG artifacts. The computation complexity of ICA is considerably lower than other EEG removal techniques. Consequently, the EEG artifact suppression with ICA technique has the capability of eliminating ocular and muscular artifacts from multi-channel EEG brain signals in real-time mode. It is critical for NFT systems to automatically suppress the EEG artifacts in real-time. Therefore, subjects are able to recognize their own current cognitive state.

\section{RESULTS}

\subsection{EEG Signal Analysis}

EEG signal analysis provides an accurate evaluation tool for measuring the cognitive performance improvement. The power spectrum of five bands (Delta $1-4 \mathrm{~Hz}$, Theta $4-8 \mathrm{~Hz}$, Alpha $8-12 \mathrm{~Hz}$, Beta $12-32 \mathrm{~Hz}$ and $\mathrm{Gamma} 32-60 \mathrm{~Hz}$ ) was calculated by utilizing MATLAB FFT function with a Hanning window and the window size is 256 samples. A relative power of each band was compared with the summation of power spectrum of all five bands. Relative power spectrum of Beta and Alpha can be used to measure the cognitive performance. The higher relative power spectrum of Theta and lower relative power of Alpha, the better cognitive abilities [12]. In each NFT session, the system recorded EEG signals at $\mathrm{AF} 3, \mathrm{AF} 4, \mathrm{O} 1$ and $\mathrm{O} 2$ for calculating the relative power spectrum of Beta and Alpha frequency bands. The relative power ratio of Beta/Alpha was utilized as an indicator of cognitive performance.

Experimental results show it is possible to train older adults to modify their amplitude of certain wave ranges using an NFT protocol. During training sessions, subjects needed to perform sustained attention by focusing the target object on the screen. From EEG data analysis, the average attention level of most participants in each training increased in most subjects. Furthermore, experimental results show the relationship between sustained attention and cognitive performance exists. The ability to sustained attention potentially leads to enhance cognitive functions. The average of task-related attention level of each participant in each training day is depicted in Figure $4 \mathrm{a}$ and $4 \mathrm{~b}$. Improved cognitive performance can be seen from upward trends of the graphs. Most participants succeeded to increase the ratio of their beta/alpha power band in both groups. In healthy group, 6 of 8 participants successfully improved their cognitive performance. Two participant, ID2 and ID4, failed to increase the ratio. In MCI group, 8 of 11 were capable of enhancing their cognitive performance. Three participants named ID10, ID16 and ID17 did not accomplish to increase the ratio.

\subsection{CANTAB Score Analysis}

The CANTAB program was utilized for subject's cognitive function assessments of pre- and post-NFT trial. In this clinical trial, all subjects required to assess different aspects of cognitive functioning: spatial working memory, pattern recognition memory, rapid visual information processing and delayed matching to sample. From a preliminary analysis, the difference of pre- and post-CANTAB score of these cognitive functions did not significantly improve in most subjects. We are currently analyzing the CANTAB scores with a statistical approach. A completed statistical analysis of the score will be reported in later stage.

\section{CONCLUSION}

The proposed neurofeedback system for attention training is aimed to encourage older adults to produce specific patterns of cortical activity in connection with an improved level of cognitive performance. After 6-week NFT intervention program, most participants in both healthy and MCI groups were able to increase the ratio of Beta/Alpha power, which indicates improved level of cognitive ability. Consequently, the results suggest that training to sustain attention using NFT methodology leads to enhance cognitive performance in both healthy and MCI groups. Moreover, EEG data analysis provides an accurate evaluation tool for measuring the cognitive performance improvement. 


\section{REFERENCES}

[1] Thomas H. Budzynski, Introduction to Quantitative EEG and Neurofeedback: Advanced Theory and Applications, Elsevier $2^{\text {nd }}$ Edition, 2009.

[2] Y. Tang, M.I. Posner, "Attention training and attention state training," Trends in Cogntive Sciences, vol 13(5), 222-227, 2009.

[3] Pondé M.P., Cruz-Freire A.C., Silveira A.A.,"Relationship Between Learning Problems and Attention Deficit in Childhood," Journal of Attention Disorder, Aug; 16(6):505-9, 2012.

[4] Duric N.S., Assmus J., Gundersen D., Elgen I.B.," Neurofeedback for the treatment of children and adolescents with ADHD: a randomized and controlled clinical trial using parental reports," BMC Psychiatry. 10;12:107, Aug 2012.

[5] Gevensleben H, Holl B, Albrecht B, et al, "Neurofeedback training in children with ADHD: 6-month follow-up of a randomised controlled trial," Eur Child Adolesc Psychiatry, 19:715-724,2010.

[6] Becerra J, Fernández T, Roca-Stappung M, et al, "Neurofeedback in Healthy Elderly Human Subjects with Electroencephalographic Risk for Cognitive Disorder," Journal of Alzheimer's Disease, vol 28, 357-367,2012.
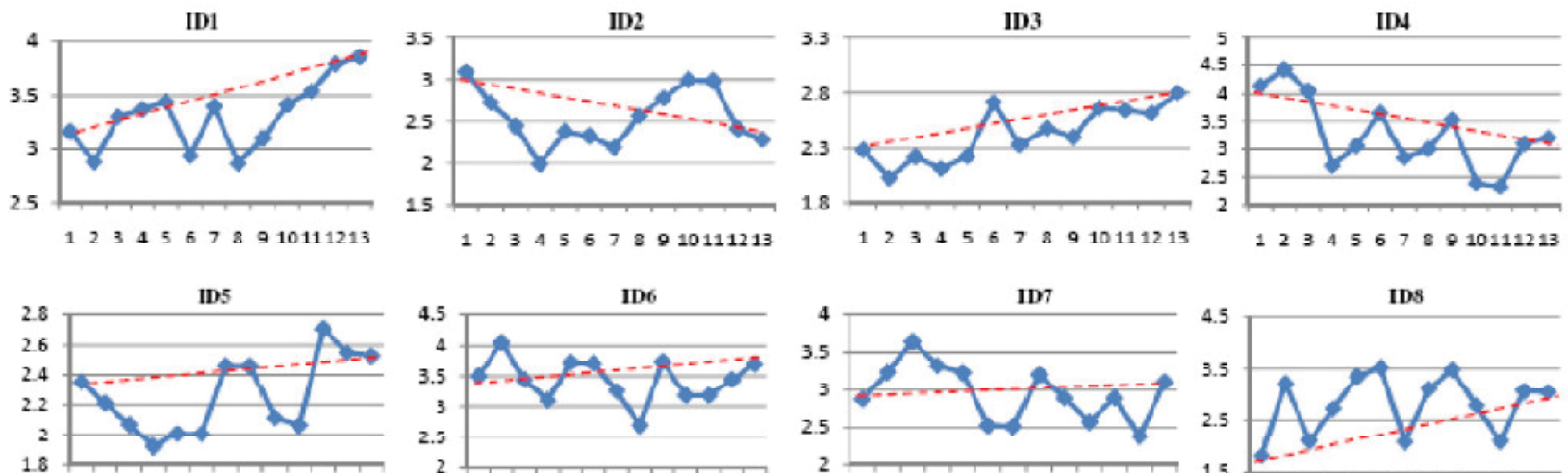

12345678910111213
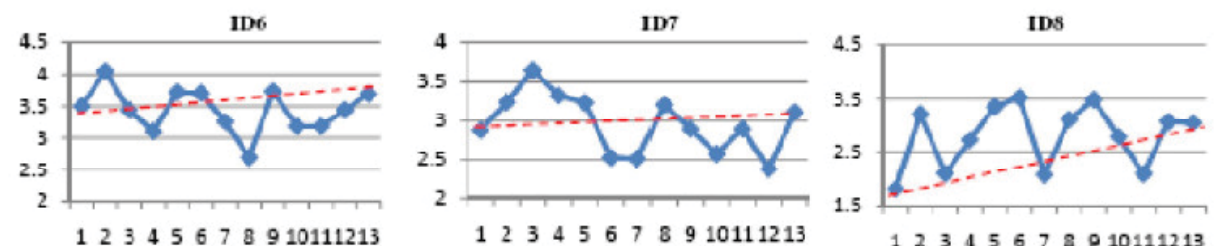

Fig 4 (a) Relative Power Ratio of Beta/Alpha in Healthy Group
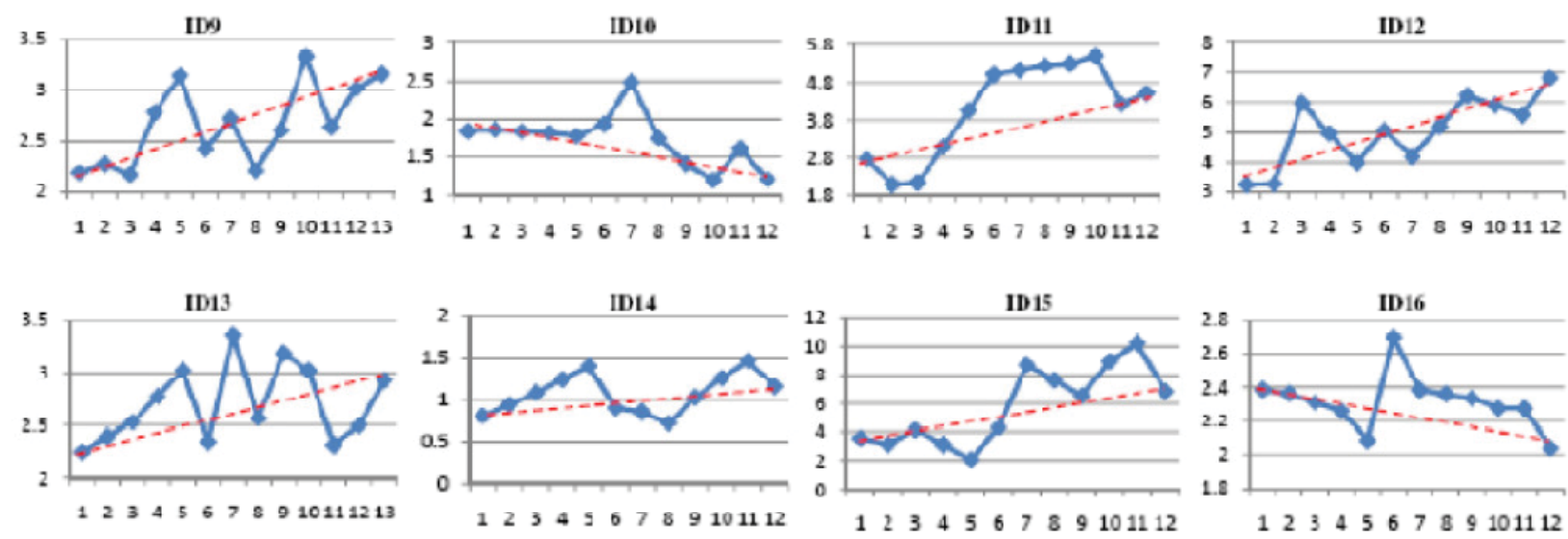
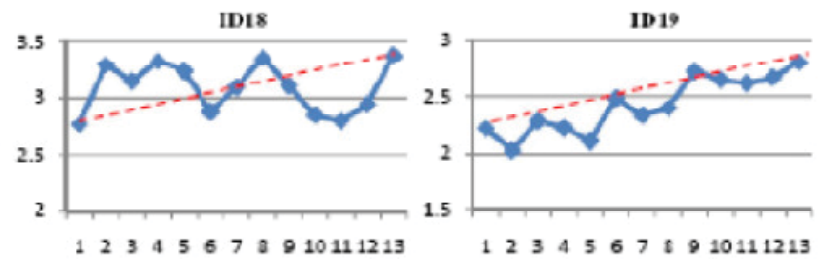

Fig 4 (b) Relative Power Ratio of Beta/Alpha in MCI Group 